Para citar este artículo: Berganza, R., Herrero, B. \& Carratalá, A. (2016). La (des)confianza de los periodistas españoles hacia las instituciones públicas a partir del estudio de factores organizacionales. Anuario Electrónico de Estudios en Comunicación Social "Disertaciones", 9(1), 24-43. Doi: dx.doi.org/10.12804/ disertaciones.09.01.2016.02

\title{
LA (DES)CONFIANZA DE LOS PERIODISTAS ESPAÑOLES HACIA LAS INSTITUCIONES PÚBLICAS A PARTIR DEL ESTUDIO DE FACTORES ORGANIZACIONALES
}

The (Dis)trust of Spanish Journalists Towards Public Institutions Based on the Study of Organizational Factors

A (des)confiança dos jornalistas espanhóis às instituições públicas a partir do estudo de fatores organizacionais

BERGANZA CONDE, Rosa. Universidad Rey Juan Carlos (España)

rosa.berganza@urjc.es

HERRERO, Beatriz. Universitat Rovira I Virgili (España)

beatriz.herrero@urv.cat

CARRATALÁ, Adolfo. Universitat de València (España)

adolfo.carratala@uv.es

Fecha de recibido: 30 de julio de 2015

Fecha de aceptado: 25 de septiembre de 2015

\section{RESUMEN}

Existe un creciente interés en la bibliografía científica por conocer las prácticas y rutinas de las y los periodistas en distintos contextos (Hanitzsch et al., 2011). La confianza que tienen estos/as profesionales en las instituciones públicas es una variable clave para comprender su cultura profesional (Hanitzsch \& Berganza, 2012; Brants, de 
Vreese, Möller \& van Praag, 2010; van Dalen, Albæk \& de Vreese, 2011), así como el interés y la confianza de la ciudadanía en la política (Cappella \& Jamieson, 1997). Este estudio pretende conocer el nivel de confianza de las y los periodistas españoles en las instituciones públicas y verificar si el tipo, alcance y propiedad del medio influyen en dichos niveles de confianza. Para ello, se llevó a cabo una encuesta a una muestra probabilística de periodistas españoles ( $n=390)$ estratificada por tipo de medio y comunidad autónoma, entre marzo de 2014 y mayo de 2015. El estudio fue desarrollado como parte del proyecto Worlds of Journalism Study. En contraste con la última medición de Hanitzsch y Berganza (2014), los resultados muestran una caída significativa en los niveles de confianza, en especial hacia el sector político y, probablemente, como consecuencia de la crisis económica. Los datos del estudio revelan diferencias parciales en los niveles de confianza respecto al tipo de medio (televisión, radio, prensa, en línea, etc.), pero niveles homogéneos entre los diferentes alcances (regional, nacional) y propiedades (pública, privada, etc.) de los medios, de modo que se sugiere avanzar hacia modelos explicativos que permitan seguir profundizando en el origen de la desconfianza de las y los periodistas españoles/as.

Palabras clave: Confianza, instituciones públicas, periodistas, medios, política.

\section{ABSTRACT}

There is an increasing interest in the literature to explore the practices and routines of journalists in different contexts (Hanitzsch et al., 2011). The trust of journalists in public institutions is a key variable to understand their professional culture (Hanitzsch \& Berganza, 2012; Brants, de Vreese, Möller \& van Praag, 2010; van Dalen, Albæk \& de Vreese, 2011), as well as the interest and trust of citizens towards politics (Cappella \& Jamieson, 1997). The goal of this paper is determining the level of trust of Spanish journalists towards public institutions and verifying if media type, reach and ownership influence these levels. As a part of the international project Worlds of Journalism Study, we surveyed a probabilistic sample $(n=390)$ of Spanish journalists, stratified by media type and region, from March 2014 to May 2015. When compared to the last survey made by Hanitzsch \& Berganza (2014), the findings show a significance decrease in the levels of trust (specially towards politics), eventually as a consequence of the economic crisis. Data also revealed partial differences in the levels of trust among journalists of different media type (TV, radio, newspaper, online, etc.), but showed homogeneous levels among professionals of different media (regional, national) or ownership (public, private, etc.). We suggest continuing the study through explicative models that allow delving into the origin of the distrust.

Keywords: Trust, Public Institutions, Journalists, Media, Politics.

\section{RESUMO}

Existe um crescente interesse na bibliografia científica por conhecer as práticas e rotinas dos jornalistas em distintos contextos (Hanitzsch et al., 2011). A confiança que têm estes profissionais nas instituições públicas é uma variável chave para compreender a sua cultura profissional (Hanitzsch \& Berganza, 2012; Brants, de Vreese, Möller 
\& van Praag, 2010; van Dalen, Albæk \& de Vreese, 2011), assim como o interesse e a confiança da cidadania na política (Cappella \& Jamieson, 1997). Este estudo pretende conhecer o nível de confiança dos jornalistas espanhóis nas instituições públicas e verificar se o tipo, alcance e propriedade do médio influem em ditos níveis de confiança. Para isto, realizou-se um questionário a uma amostra probabilística de jornalistas espanhóis ( $\mathrm{n}=390$ ) estratificada por tipo de meio e comunidade autónoma, entre março de 2014 e maio de 2015. 0 estudo foi desenvolvido como parte do projeto Worlds of Journalism Study. Em contraste com a última medição de Hanitzsch e Berganza (2014), os resultados mostram uma queda significativa nos níveis de confiança em especial ao setor político e provavelmente, como consequência da crise económica. Os dados do estudo revelam diferenças parciais nos níveis de confiança respeito ao tipo de meio (televisão, rádio, imprensa, on-line, etc.), mas níveis homogêneos entre os diferentes alcances (regional, nacional) e propriedades (pública, privada, etc.) dos meios, de modo que se sugere avançar a modelos explicativos que permitam seguir aprofundando na origem da desconfiança dos jornalistas espanhóis.

Palavras-chave: Confiança, instituições públicas, jornalistas, meios, política.

\section{El papel de los medios de comunicación en la desafección ciudadana hacia las instituciones públicas}

La difícil coyuntura económica que atraviesa España desde 2008, como consecuencia de la crisis financiera de carácter internacional, se ha visto agravada por las debilidades del modelo productivo en el que el país había basado su crecimiento en los años previos a la crisis, y por la continua sucesión de escándalos políticos -en su mayoría vinculados con delitos de corrupción- que afectan a los diferentes partidos políticos y golpean a todos los niveles de la administración. Este panorama influye de manera evidente en la percepción que la ciudadanía tiene de las instituciones públicas, tal y como recogen los trabajos demoscópicos más recientes.

Según los datos del Barómetro número 3082 de mayo de 2015 del Centro de Investigaciones Sociológicas (cis), la ciudadanía percibe la situación política actual como problemática, en gran medida debido a la corrupción política. La situación no es exclusiva de España, ya que, de acuerdo con el Eurobarómetro que recoge los datos del periodo 2003-2013, la desconfianza de la ciudadanía hacia los partidos políticos creció notablemente desde 2008 en los países del sur de Europa que comparten, con España, un agravamiento de los problemas políticos y económicos, especialmente Portugal y Grecia. A diferencia de lo que ocurre con los países del norte, en estos Estados los últimos índices de desconfianza registrados hacia los partidos políticos, el Gobierno y el Parlamento son los más altos desde 2003. Similar recorrido siguen las líneas que representan la insatisfacción ciudadana con la democracia; como señala Mair (2006), nunca antes en la historia de la Europa de posguerra se había tenido a los gobiernos y a los líderes políticos en tan baja estima. 
El descenso de la confianza que las/los ciudadanas/os depositan en las instituciones que gobiernan y gestionan la convivencia de las sociedades (Parlamento, Gobierno, partidos políticos, justicia/tribunales, policía, políticas/os) denota un claro descrédito que las/los representadas/os tienen sobre la capacidad de acción de estos agentes. La confianza, como indica Misztal (1996), implica proyectar esperanza en el porvenir, presumir que la actuación de una determinada persona o institución responderá a nuestros deseos. La confianza institucional, pues, supone tener una elevada expectativa de que una institución actuará en el futuro de manera satisfactoria (Hudson, 2006). Cuando esa creencia falla, la desconfianza domina la percepción ciudadana de los poderes públicos, afectando el bienestar de las sociedades. Como resultado, la/el ciudadana/o desarrolla una actitud cínica que dominará su percepción de la realidad pese a que no haya evidencias en las que sustentarla, una disposición que se caracteriza por entender que el sistema político es corrupto y que sus representantes son agentes partidistas y maquiavélicos a quienes no les interesa el bien común ni tampoco gobernar, sino únicamente salir victoriosos de todo proceso y actividad en los que se vean involucrados (Capella \& Jamieson, 1977).

Los medios de comunicación han sido señalados como posibles factores explicativos de por qué la ciudadanía se aleja de sus representantes. Es decir, la cobertura periodística desfavorable de las instituciones públicas y de los actores y actrices políticos/as contribuiría a un creciente desapego de los individuos respecto de estos agentes. Así lo apunta, por ejemplo, la llamada teoría de la media malaise (Robinson, 1976), que sostiene que un enfoque predominantemente adverso y anti-institucional tiene claros efectos en las/los receptoras/es: "un tratamiento negativo y altamente crítico de los medios respecto a las instituciones políticas y los políticos ha dado lugar a un aumento de las opiniones y percepciones negativas de los objetos políticos" (Uriarte, 2002, p. 364).

En la misma línea apunta la teoría conocida como espiral del cinismo. De acuerdo con este planteamiento, el estilo y el contenido del periodismo político puede fomentar el aumento del cinismo y de la desconfianza de la audiencia hacia las instituciones si, por ejemplo, las noticias responden de forma persistente a un patrón de negatividad. Además, recuerdan Capella y Jamieson (1997), la influencia de los medios es mayor en aquellos temas en los que nuestra experiencia directa es escasa. Por ello, dado que habitualmente la ciudadanía no goza de un contacto estrecho con sus líderes, sus campañas y sus actuaciones políticas, es la prensa la encargada de asumir esa mediación. Estos autores aseguran que el enfoque estratégico propio del periodismo contemporáneo favorece precisamente una lectura de la actualidad política en la que quienes gestionan lo público aparecen representados como actores y actrices interesados/as únicamente en su propio beneficio, deseosos de ganar cuanto puedan y hacerlo, además, a toda costa. Este encuadre sería el causante de que las y los líderes políticas/os -y, por extensión, las instituciones por ellas/os gobernadas - apenas gocen de la confianza de la ciudadanía.

Sin embargo, el alcance de esta influencia podría verse limitado por el escepticismo de las/los ciudadanas/os hacia los actores mediáticos. Así, de igual modo que la investigación ha revelado que la desconfianza de los individuos hacia los medios de comunicación favorece una moderación de los efectos de la agenda-setting (Tsfati, 2003), esa misma actitud podría modular la influencia mediática sobre el cinismo de la audiencia hacia las instituciones.

En cualquier caso, las investigaciones realizadas en este ámbito no corroboran un modelo de influencia esquemático y lineal en el que los contenidos mediáticos determinen por completo la confianza ciudadana en las instituciones. El impacto de las noticias en los niveles de confianza parece depender de un conjunto más complejo de variables, más allá del nivel crítico de las informaciones, que hace necesario tener en consideración el tipo de medio, el contenido del mensaje y los niveles de confianza previos (Van Dalen, Albæk \& De Vreese, 2011). En las 
revisiones posteriores a la formulación de ese modelo se ha hecho hincapié en señalar que el cinismo, que cada vez más se identifica entre la ciudadanía, no se inicia con el relato que los medios de comunicación ofrecen de la actualidad. En el estudio de este fenómeno es preciso observar con atención qué percepciones del sistema político dominan entre quienes son los encargados de elaborar ese mensaje y, por lo tanto, dirigir la atención al tipo de relación que quienes ejercen el periodismo mantienen con los actores y actrices políticos/as y a los valores que caracterizan esa interacción (Van Dalen, Albæk \& De Vreese, 2011; Brants et al., 2010).

Dadas las posibles conexiones entre la actitud de quienes ejercen el periodismo y el modo en que realizan su trabajo, resulta razonable considerar que la producción de coberturas dominantemente negativas sobre la actualidad política se relacione con la percepción que las y los periodistas tienen de los actores o actrices políticos/as y de las instituciones que estos gobiernan. Por ello, las dos preguntas de investigación a las que intentaremos dar respuesta con este trabajo se refieren a lo siguiente:

PI1. ¿Qué nivel de confianza tienen las y los periodistas españoles en las principales instituciones políticas (Gobierno, Parlamento, partidos políticos y políticas/os en general)?

PI2. ¿Varía esta confianza en función de las características del medio en el que trabajan?

\section{La organización mediática como factor de influencia en los niveles de confianza de las y los periodistas}

Son múltiples los factores que desde la academia se señalan como las claves para comprender los niveles de confianza. Algunos de estos factores se engloban en lo que determinados autores (Mishler \& Rose, 2001) han identificado como las teorías institucionales de la confianza política; es decir, las perspectivas que defienden que la confianza es endógena a la propia política y, por tanto, consecuencia de la actuación de los propios actores e instituciones, por ejemplo en el terreno de la economía, de la calidad de la democracia o de la libertad de prensa. Frente a esta explicación encontramos las teorías culturales, que apuntan a un origen exógeno de la confianza política, entendiéndola como una extensión de la confianza interpersonal. No obstante, unos factores y otros no son excluyentes. De hecho, investigaciones previas (Hanitzsch \& Berganza, 2012) señalan que son de diverso orden los factores que impactan en la confianza que las y los periodistas tienen sobre las instituciones públicas: el modo en que actúan dichas entidades, la confianza interpersonal, la cultura periodística o la propiedad del medio.

Este último factor, que se correspondería con el nivel organizacional, demostró, por ejemplo, que las y los periodistas que trabajan en medios de comunicación de titularidad estatal comparten mayores niveles de confianza en las instituciones. El hallazgo prueba que el estudio de los factores vinculados con la organización mediática es decisivo en la investigación sobre comunicación, tal y como apuntan otros trabajos que certifican que las características del medio son determinantes tanto para entender la producción de noticias (Cook, 1996; Gans, 2003; Weaver \& Loffelholz, 2008) y el contenido emitido (Shoemaker \& Reese, 1996), como para interpretar las funciones y los roles que las y los periodistas consideran que los medios deben desarrollar en sociedad (Zhu et al., 1997). Asimismo, las variables relacionadas con la organización mediática (por ejemplo, el que un medio de comunicación tenga una orientación popular o se considere un medio de prestigio) se han revelado significativas en el 
estudio sobre por qué se producen desajustes entre el rol ideal con el que se identifican las/los periodistas y el rol que finalmente llevan a la práctica en su trabajo (Mellado \& van Dalen, 2014).

El mesonivel o nivel organizacional incluye tanto el entorno de la redacción, como el contexto gerencial y económico de la producción de noticias (Reich \& Hanitzsch, 2013) en el que entran en juego, además de la propiedad, el tamaño, el radio de cobertura y el tipo de medio de comunicación. Así, si como hemos visto, las teorías institucionales y culturales apuntan hacia otras variables que afectan la confianza de los profesionales de la información, el nivel organizacional se mantiene como un interesante objeto de estudio pues, como desvelan Hanitzsch y Berganza (2012), este nivel da cuenta del 7,5\% de la varianza de la confianza de las y los periodistas en las instituciones.

Esta cifra, que fue extraída de un estudio comparativo internacional y que ha de ser tenida en cuenta, nos lleva a profundizar en el conocimiento sobre la influencia que juega el aspecto organizacional en la confianza que las y los periodistas españoles depositan en las instituciones públicas, tomando en consideración el tipo, el alcance y la propiedad de los medios en los que trabajan.

\section{Planteamiento del estudio}

En lo que respecta a los tipos de medios de comunicación, el panorama mediático español se ha ido transformando desde el inicio de la crisis económica. Muestra de ello es que, entre 2008 y 2013, la estimación de empleos perdidos en el sector de los medios de comunicación en España es de 9471 y se calcula que 284 medios han desaparecido. Estos datos de la Asociación de la Prensa de Madrid (APM, 2013) hacen referencia a todo tipo de trabajadores/as de los medios de comunicación, no exclusivamente a periodistas. La desagregación resulta imposible dado que España no cuenta con un registro de periodistas en activo. Sin embargo, quizás lo más interesante de la crisis de los medios ha sido la emergencia de nuevos medios de comunicación nativos digitales creados por periodistas, muchos/as de los/as cuales han perdido su empleo durante la crisis (APM, 2013, p. 85). Debido a la propia estructura del medio, más ligera, o a las propias experiencias profesionales de las y los periodistas que conforman las redacciones de este tipo de organizaciones, es posible que su confianza en las instituciones públicas varíe. De hecho, un estudio realizado a partir de una muestra de periodistas de Europa y Estados Unidos encontró niveles más altos de negatividad en los medios en línea comparados con los de los medios impresos (Quandt, 2008), lo cual, entre otros factores, consideramos que podría estar relacionado con la confianza de los y las profesionales hacia sus instituciones.

Asimismo, es evidente que la crisis no ha afectado por igual a los distintos medios de comunicación en España. Si bien desde 2008 la facturación ha descendido en todos ellos, la reducción de los ingresos agregados entre 2008 y 2012 varía por medios de comunicación. Por ejemplo, en la radio la pérdida asciende hasta un $21 \%$, mientras que en televisión es de un 31 \% y en prensa es de un 30 \% (APM, 2013). La diferencia existente de los entornos profesionales según se trabaje en un medio de comunicación o en otro nos lleva a proponer la siguiente hipótesis:

H1. Existen diferencias significativas en los niveles de confianza de las y los periodistas hacia las instituciones políticas (Gobierno, Parlamento, partidos políticos y políticas/os en general) en función del tipo de medio en el que trabajan (televisión, radio, prensa, en línea, etc.). 
Con referencia al alcance de los medios de comunicación, Hanitzsch y Berganza (2012) sugieren que la libertad de prensa y, por tanto, el grado de autonomía garantizado por las instituciones públicas a los medios de comunicación, puede modelar las percepciones que las y los periodistas tienen de dichas instituciones. De esta manera, las instituciones políticas, organizadas en diferentes niveles de alcance de gobierno a lo largo del territorio español, pueden ejercer una presión de diferente intensidad sobre los medios de comunicación que informan sobre sus actividades. En este sentido, merece la pena medir si existen diferencias en los niveles de confianza de las y los periodistas dependiendo del alcance del medio en el que trabajan, debido a que precisamente esa variable determina en gran medida las instituciones sobre las que informan; es decir, sobre qué nivel de la administración pública (municipal, autonómico o estatal) concentran su cobertura.

Además, es interesante anotar que los medios de comunicación de menor alcance territorial (municipal y regional) suelen depender más directamente de las inversiones publicitarias de las instituciones públicas, lo cual puede ejercer una influencia directa en los niveles de percepción de autonomía de las y los periodistas (Reich \& Hanitzsch, 2013) y, como consecuencia, podría alterar su confianza en las instituciones. Igualmente, el alcance del medio suele ir relacionado con su tamaño. Así, un medio que cubre noticias locales habitualmente presenta un menor tamaño que aquel que elabora informaciones de ámbito nacional, lo cual supone variaciones en el entorno organizacional que podrían afectar la confianza de las y los informadoras/es. Por lo tanto, planteamos esta segunda hipótesis:

H2. Existen diferencias significativas en los niveles de confianza de las y los periodistas hacia las instituciones públicas en función del alcance del medio (local, regional, nacional).

Por su parte, en lo que a la propiedad de los medios de comunicación se refiere, esta se presenta en estudios anteriores como uno de los principales factores que determinan la producción de noticias en el nivel organizacional (Donohue, Olien \& Tichenor, 1985; Reich \& Hanitzsch, 2013; Weaver et al., 2007; Zhu et al., 1997). No obstante, no existe un consenso en la bibliografía académica sobre si las y los periodistas de los medios de comunicación privados tienen un menor nivel de confianza en las instituciones (McManus, 1994) o no (Hanitzsch \& Berganza, 2012) que las y los periodistas de los medios públicos. Reich y Hanitzsch (2013, p. 147) afirman que, en su investigación sobre las influencias percibidas por periodistas de 18 países, las y los profesionales de los medios públicos mostraban una menor autonomía que sus compañeros/as de los medios privados, lo cual podría estar relacionado con los niveles de desconfianza en las instituciones políticas.

En Europa Occidental, la propiedad de los medios de comunicación se divide habitualmente entre medios públicos, estatales y privados ${ }^{1}$ (Hanitzsch \& Mellado, 2011), aunque en España el número de medios gestionados directamente por las instituciones públicas (es decir, estatales) es muy pequeño, según se desprende del listado de medios confeccionado para esta investigación a partir de la exhaustiva revisión de la información facilitada tanto por la Asociación de la Prensa de Madrid (APM) como por la Agenda de la Comunicación y tras haber descartado

1 La diferencia entre estatal y público a menudo es confusa. La cuestión radica en si el medio es propiedad directa del Gobierno (sea nacional, regional o municipal), en cuyo caso es estatal, o si, por el contrario, el medio lo dirige una entidad legalmente independiente que recibe subsidios del Gobierno, sufragados mediante impuestos o suscripciones obligatorias. En este último caso se trataría de un medio público. 
aquellos que estaban inactivos en el momento del estudio. En cualquier caso, según afirman Hanitzsch y Berganza (2012), dentro del nivel organizacional, la titularidad de los medios explica aproximadamente dos tercios de las variaciones de la confianza de las y los informadores en las instituciones públicas. Ello nos lleva a sugerir una tercera hipótesis:

H3. Existen diferencias significativas en los niveles de confianza de las y los periodistas hacia las instituciones públicas en función de la propiedad del medio (pública, privada, mixta, estatal)

\section{Método de análisis}

\section{Muestra y procedimiento}

El presente trabajo se realizó en el marco de un proyecto internacional, Worlds of Journalism Study (WJS, www. worldsofjournalism.org), y fue financiado por la Universidad de Münich, Alemania, y complementado con fondos del proyecto del Ministerio de Economía y Competitividad (CSO2013-44874-R). Para su realización se aplicó una encuesta nacional a periodistas españolas/es con la metodología propuesta y validada por las y los investigadoras/es del proyecto WJS. El periodo de recolección de datos tuvo lugar entre marzo de 2014 y mayo de 2015. Los cuestionarios, que se realizaron por teléfono a una muestra representativa de periodistas, fueron aplicados por encuestadoras/es que habían recibido entrenamiento previo por parte del equipo investigador del país analizado.

Específicamente se realizó un muestreo polietápico, que incluye selección por conglomerados (clúster) y estratificación por tamaño (grande/pequeño), tipo de medio (periódico/ agencia/ radio/ televisión/ revista/medio en línea) y comunidad autónoma. En vista de la imposibilidad de conseguir un listado nacional de periodistas en activo en España, se calculó el tamaño de la población a partir del punto promedio del rango propuesto por Díaz-Nosty para el año 2010 ( $\left.n_{1}=22550\right)$, restándole a esta cifra la estimación del $20 \%$ de despidos hecha por la Asociación de la Prensa de Madrid (APM, 2013). Como resultado de esta operación, calculamos la población de nuestro estudio $\left(n_{2}=18000\right)$ y el tamaño muestral de la encuesta $(n=390)$ para un $95 \%$ de confianza y un margen de error del $5 \%$.

El procedimiento de selección de la muestra se realizó en primer lugar por racimos, tomando como referencia los medios de comunicación como unidad muestral agregada. Se elaboró un listado $\left(n_{3}=382\right)$ que incluyó aquellos referenciados en el informe de la APM (2013) y en la Agenda de la Comunicación, elaborada por el Gobierno español. Tras el proceso de estratificación (tabla 1), se seleccionaron aleatoriamente 117 medios (tasa de respuesta efectiva del 62,9\%, en total se consultaron 186 medios). En los medios considerados pequeños se encuestó a 3 personas y 5 en los grandes ${ }^{2}$. La muestra definitiva fue de 390 periodistas y la tasa de respuesta efectiva, del 82,28\% (en total se consultó a 474 profesionales).

2 En cada medio se escogió al menos a un/a redactor/a, un/a periodista en puestos de mando intermedio (junior) y un/a directivo/a (senior). 
Tabla 1. Distribución de medios seleccionados por tamaño y comunidad autónoma

\begin{tabular}{|c|c|c|c|c|c|c|c|c|c|c|c|c|}
\hline \multirow{3}{*}{ CCAA } & \multicolumn{12}{|c|}{ Tipo de medio } \\
\hline & \multicolumn{2}{|c|}{ Periódicos } & \multicolumn{2}{|c|}{ Agencia } & \multicolumn{2}{|c|}{ Radio } & \multicolumn{2}{|c|}{ Televisión } & \multicolumn{2}{|c|}{ Revistas } & \multicolumn{2}{|c|}{ Digitales } \\
\hline & G & $\mathbf{P}$ & G & $\mathbf{P}$ & G & $\mathbf{P}$ & G & $\mathbf{P}$ & G & $\mathbf{P}$ & G & $\mathbf{P}$ \\
\hline Andalucía & & 6 & & & & & & 3 & & & & 5 \\
\hline Aragón & & 2 & & 1 & & 1 & 1 & & & & & \\
\hline Asturias & & & & & & 1 & & 1 & & & & \\
\hline Baleares & & 2 & & & & 1 & & & & & & \\
\hline Canarias & & & & & & & & 1 & & & & 1 \\
\hline Cantabria & & 1 & & & & & & & & & & \\
\hline Castilla La Mancha & & 2 & & & & & 1 & 2 & & & & \\
\hline Castilla y León & & 2 & & & & & & & & & & 3 \\
\hline Cataluña & & 10 & & & & 1 & & 3 & 1 & & & 4 \\
\hline Comunidad Valenciana & 1 & 7 & & & & 1 & & & & & & 2 \\
\hline Extremadura & & & & & & & & & & & & \\
\hline Galicia & 1 & 3 & & & & 1 & & 1 & & & & 1 \\
\hline Madrid & 2 & 4 & 1 & & 2 & 3 & 2 & 1 & & 3 & 7 & 2 \\
\hline Murcia & & & & & & & & & & & & \\
\hline Navarra & 1 & 1 & & & & 1 & 1 & 1 & & & & \\
\hline País Vasco & & 3 & & 1 & & 1 & & 2 & & & & 1 \\
\hline La Rioja & & 1 & & & & 1 & & & & & & \\
\hline Ceuta y Melilla & & 1 & & & & & & & & & & \\
\hline Total medios & 5 & 45 & 1 & 2 & 2 & 12 & 5 & 15 & 1 & 3 & 7 & 19 \\
\hline
\end{tabular}

Leyenda: G: medio grande/ P: medio pequeño

Fuente: elaboración propia.

\section{Medidas}

Con el fin de medir la confianza de las y los periodistas en las instituciones políticas españolas, se construyó un índice de confianza (1-5), en donde 1 representa "ninguna confianza" y 5 "total confianza". El índice se construyó específicamente a partir del promedio de las puntuaciones de 4 instituciones: a) el Parlamento, b) el Gobierno, c) los partidos políticos y d) los políticos en general. La validez de este constructo fue constatada por medio de un análisis factorial exploratorio (AFE), el cual reveló la unidimensionalidad del mismo. Este análisis permite la identificación de factores subyacentes en una serie de variables (Pérez-Gil, Chacón \& Moreno, 2000; Macía, 2010; Igartua, 2006). Específicamente, 
el AfE sugirió una estructura latente de un solo componente, explicando el 61,57 \% de la varianza para el conjunto de los 4 indicadores. La prueba Kaiser-Meyer Olkin sugirió que la muestra era factorizable (кмо $=0,688)$. Con respecto a la consistencia interna, nuestro índice obtuvo también una fiabilidad adecuada (Alfa de Cronbach=0,787), siguiendo el valor mínimo ideal $(>0,70)$ sugerido por Cronbach (1951) y Hayes (2005).

\section{Análisis}

Las encuestas realizadas fueron revisadas por el equipo investigador y posteriormente codificadas gracias al programa sPss (versión 21). Seguidamente, se realizó un análisis preliminar y exploratorio de los datos para detectar los campos vacíos y conocer la fiabilidad de las medidas. Para verificar las hipótesis del estudio se llevó a cabo un análisis descriptivo con el fin de conocer las medidas centrales y de dispersión, y luego un análisis inferencial con pruebas de diferencia de medias $t$ Student y Anova (One-way). Este último análisis se hizo con un bootstrap de 1000 muestras (95\% de nivel de confianza y método de sesgos corregidos).

\section{Resultados}

La muestra de periodistas encuestada $(n=390)$ pertenece fundamentalmente a medios impresos o agencias de noticias $(47,4 \%)$ y, en menor medida, a radio o televisión $(29,7 \%)$ o medios en línea $(22,9 \%)$. La gran mayoría trabajan para medios locales o regionales $(75,9 \%)$ y el resto para medios nacionales $(24,1 \%)$. Con respecto a la propiedad del medio, sólo el $14,9 \%$ de las y los entrevistadas/os pertenecen a medios públicos o estatales, mientras que el 85,1\% están vinculados a medios privados. La mayoría son hombres (59 \%) con una edad promedio de 39,5 años. Casi todos cuentan con estudios universitarios $(72,3 \%)$, de máster $(22,6 \%)$ o de doctorado $(1,8 \%)$; aunque se encontraron algunos que apenas habían finalizado la educación media (3,3\%). Según su posición en la organización para la que trabajan, $65,1 \%$ fueron catalogados como redactores o redactoras, 22,8\% como mandos intermedios (junior) y 12,1\% como directivos o directivas (senior). La mayoría trabajan a tiempo completo para el medio (85,6\%), salvo en algunos casos en los que su afiliación era por media jornada (5,1\%) o como freelancer (9,2\%). Sólo un 32,8\% manifestó trabajar para una única fuente periodística especializada y apenas un $26 \%$ confesó profesar algún tipo de credo o religión.

El nivel de confianza de las y los periodistas hacia las instituciones políticas en España (Parlamento, Gobierno, partidos políticos y políticos en general) obtuvo una puntuación media ( $\mathrm{M}=2,29, \mathrm{DE}=0,70)$ dentro de la escala del 1 al 5 (siendo el 5 máxima confianza). Esta puntuación aumenta $(M=2,60, D E=0,60)$ cuando se incluyen en la variable otras instituciones (sistema judicial, policía, militares, sindicatos, líderes religiosos y medios de comunicación informativos). Por tanto, en general, las y los periodistas desconfían en mayor medida de las instituciones políticas que del resto de instituciones del país (con la excepción de los líderes religiosos y los sindicatos).

Esta valoración general hacia las instituciones está significativamente por debajo, $t(482)=-5,740, p<0,001$, Bootcl $_{95}=-0,61$ a $-0,31, d=0,67$, de la obtenida en una encuesta anterior realizada dentro del marco de wus ( $\mathrm{M}=2,75$, $D E=0,67)$ y que fue finalizada en 2011. En ella se incluyeron estas mismas medidas (Hanitzsch \& Berganza, 2014) ${ }^{3}$ La tabla 2 muestra los niveles de confianza específicos para cada institución.

3 En el artículo de Hanitzsch y Berganza de 2012 se estudia la confianza pública de las y los periodistas a partir de seis instituciones: Parlamento, partidos políticos, Gobierno, sistema judicial, policía y las/los políticas/os en general. Sin embargo, estos autores no utilizaron el mismo sistema ni la misma escala de medición. 
Tabla 2. Niveles de confianza (escala 1-5) de las/los periodistas hacia las instituciones en España

\begin{tabular}{|l|c|c|}
\hline \multicolumn{1}{|c|}{ Institución } & M & 1,00 \\
\hline El Parlamento & 2,81 & 0,97 \\
\hline El Gobierno & 2,24 & 0,80 \\
\hline Los partidos políticos & 1,98 & 0,80 \\
\hline Los políticos en general & 2,11 & $\mathbf{0 , 7 0}$ \\
\hline Índice (4 indicadores) & $\mathbf{2 , 2 9}$ & 0,93 \\
\hline El sistema judicial & 2,96 & 1,02 \\
\hline La policía & 3,31 & 1,17 \\
\hline Los militares & 3,03 & 0,94 \\
\hline Los sindicatos & 2,32 & 0,98 \\
\hline Los líderes religiosos & 2,02 & 0,76 \\
\hline Los medios de comunicación informativos & 3,32 & $\mathbf{0 , 6 0}$ \\
\hline Índice (10 indicadores) & $\mathbf{2 , 6 0}$ & \\
\hline
\end{tabular}

Fuente: elaboración propia.

Las instituciones políticas peor valoradas fueron los partidos políticos ( $\mathrm{M}=1,98, \mathrm{DE}=0,80)$ y los políticos en general ( $\mathrm{M}=2,11, \mathrm{DE}=0,80$ ). Lo anterior sugiere que la principal fuente de desafección hacia la política parece estar generada por la desconfianza hacia los mismos políticas/os y que la crisis económica ha evidenciado la responsabilidad fallida de estos actores y actrices institucionales.

Respecto al resto de instituciones presentes en el estudio, las peor valoradas son los líderes religiosos, seguidas de los sindicatos. Las mejores, los medios de comunicación informativos, la policía y los militares.

Las pruebas revelan que, tomadas en general como índice agregado de las cuatro instituciones políticas (Gobierno, Parlamento, partidos y políticas/os en general), las diferencias entre los niveles de confianza de las y los periodistas que ejercen su profesión en distintos tipos de medios no son estadísticamente significativas $F(2,386)=2,613 p=0,07$. Sin embargo, resulta interesante observar la tendencia: los mayores índices de confianza los poseen quienes trabajan en medios audiovisuales (radio y televisión), seguidos de los de medios impresos. Los que más desconfían son las y los profesionales de los medios en línea. Un examen detallado de las puntuaciones por cada indicador (tabla 3) mostró que las diferencias encontradas en la confianza hacia el Gobierno sí fueron estadísticamente significativas y apuntan de nuevo a una mayor desconfianza de las y los periodistas en línea hacia las instituciones políticas ${ }^{4}, F$ de Welch $(2,211,324)=5,500 p<0,01$, (véase a este respecto la Figura 1 ). Las pruebas post hoc mostraron que la confianza en el Gobierno es mayor entre las/los periodistas de televisión y de

4 Adicionalmente, hallamos que las diferencias en los niveles de confianza hacia el sistema judicial (no incluido en el índice de instituciones políticas), también fueron significativas $(F(2,383)=5,557 p<0,01)$. Se encontró que las/los periodistas de medios impresos y agencias $(\mathrm{M}=3,11, \mathrm{DE}=0,94)$ puntuaron significativamente por 


\section{ESTUDIOS}

Figura 1. Variación de los niveles de confianza según el tipo de medio

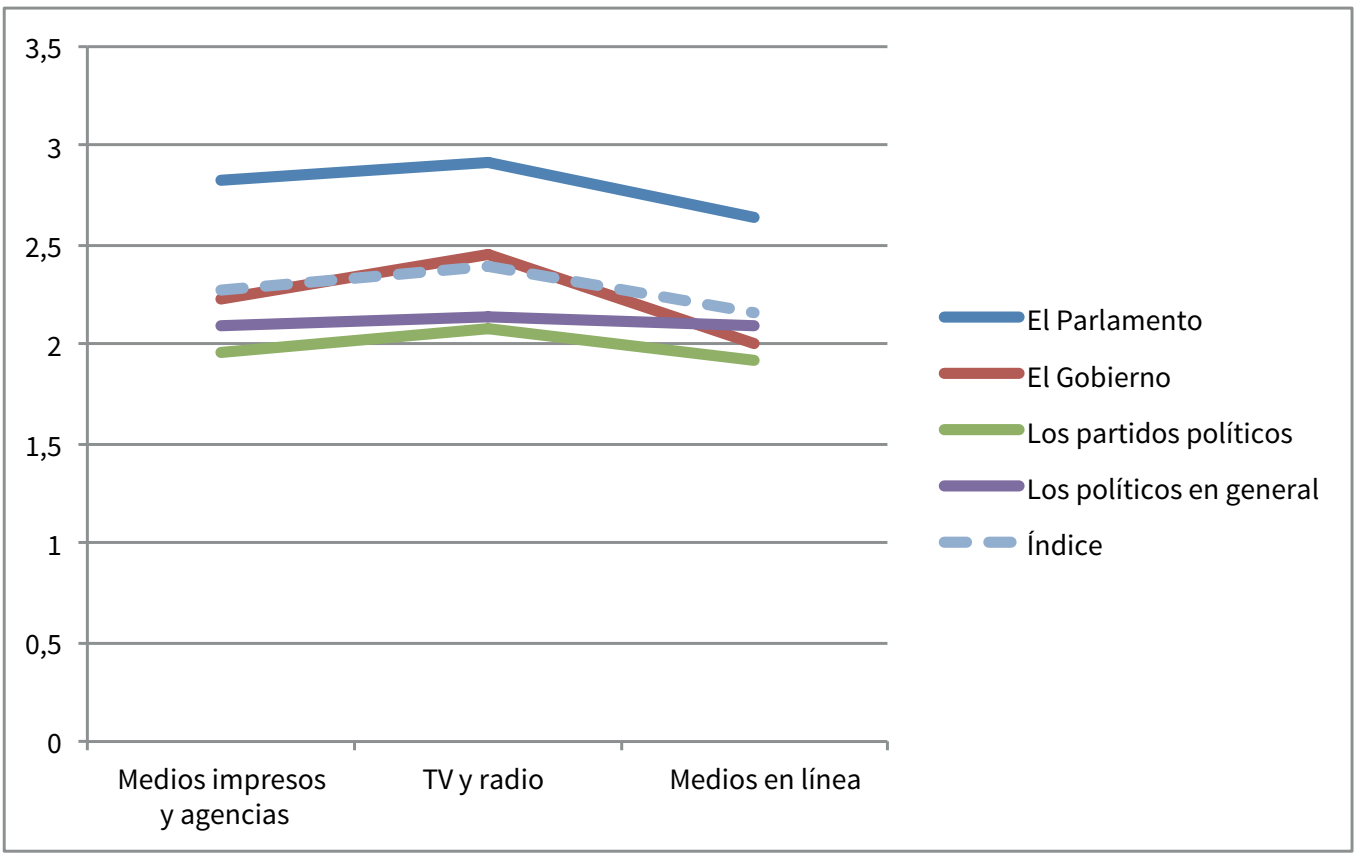

Fuente: elaboración propia.

Tabla 3. Niveles de confianza según el tipo de medio

\begin{tabular}{|l|c|c|c|c|c|c|}
\multicolumn{2}{r}{ Nivel de confianza } & \multicolumn{2}{c}{ Medios impresos y agencias } & \multicolumn{2}{c}{ TV y radio } & \multicolumn{2}{c|}{ Medios en línea } \\
\hline El Parlamento & $\mathbf{M}$ & $\mathbf{D E}$ & $\mathbf{M}$ & $\mathbf{D E}$ & $\mathbf{M}$ & DE \\
\hline El Gobierno & 2,83 & 1,01 & 2,91 & 1,04 & 2,64 & 0,94 \\
\hline Los partidos políticos & 2,23 & 0,93 & 2,45 & 1,04 & 2,00 & 0,88 \\
\hline Los políticos en general & 1,96 & 0,76 & 2,08 & 0,86 & 1,92 & 0,82 \\
\hline Índice & 2,09 & 0,78 & 2,14 & 0,82 & 2,10 & 0,83 \\
\hline
\end{tabular}

Fuente: elaboración propia.

radio $(M=2,45, D E=1,04)$ que en las/los profesionales de los medios en línea $\left(M=2,00, D E=0,88 ; B_{0 o t C l}=0,19\right.$ a 0,79; $d=0.47$ ). El tamaño de esta diferencia podría ser considerado como medio (Cohen, 1977). Podemos afirmar, por

encima que las/los periodistas de medios en línea ( $\mathrm{M}=2,72, \mathrm{DE}=0,95), \mathrm{BootCl}_{95}=0,10$ a 0,67; $d=0.41$. Esta es una diferencia que puede ser calificada como de tamaño medio. 


\section{ESTUDIOS}

tanto, que las/los periodistas que trabajan en la red son los más escépticos hacia las instituciones políticas en general y de manera especial hacia el Gobierno.

Asimismo, se hallaron diferencias significativas, aunque bajas, entre los niveles de confianza de las/los profesionales de los medios en línea al ser comparados con quienes ejercen la profesión en medios impresos y agencias $(\mathrm{M}=2,23, \mathrm{DE}=0,99), \mathrm{BootCl}_{95}=0,01$ a 0,$46 ; d=0.25$. Todos estos datos nos permiten afirmar que la hipótesis 1 , que indicaba que el tipo de medio en el que trabajan las y los periodistas implicaría diferencias en sus niveles de confianza hacia las instituciones políticas, se confirma parcialmente.

Cuando comparamos el nivel de confianza en las instituciones de las/los periodistas (figura 2) de medios locales o regionales $(M=2,27, D=0,70)$ con el de los de medios nacionales $(M=2,33, D E=0,72)$ observamos que las diferencias no son significativas, $\mathrm{t}(386)=-0,638, \mathrm{p}=0,52, \mathrm{BootCl}_{95}=-0,22$ a 0,11 , lo cual no da soporte empírico a la hipótesis 2. Lo mismo sucede cuando hacemos esta comparación en cada uno de los 4 indicadores específicos (tabla 4)5.

Figura 2. Variación de los niveles de confianza según el alcance del medio

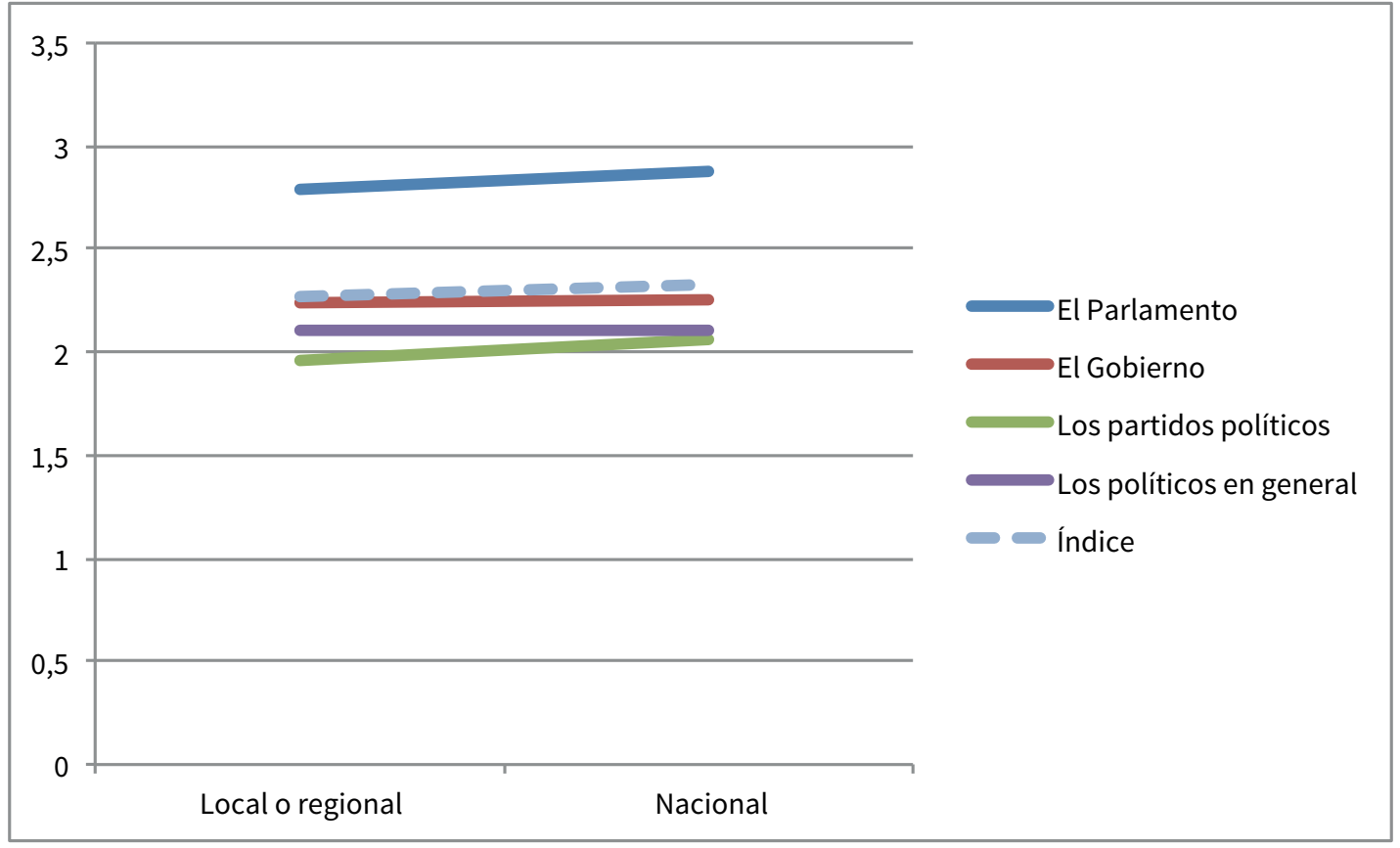

Fuente: elaboración propia.

5 Cuando ampliamos el análisis a otros indicadores, encontramos que la confianza en la policía es significativamente mayor, $\mathrm{t}(382)=-2,688, \mathrm{p}<0,01, \mathrm{BootCl}_{95}=-0,56 \mathrm{a}-0,09, d=0,31$, entre las/los periodistas de medios nacionales ( $\mathrm{M}=3,55, \mathrm{DE}=0,98)$, con respecto a las/los periodistas de medios locales $(\mathrm{M}=3,24, \mathrm{DE}=1,02)$. No obstante, el tamaño del efecto de esta diferencia es bajo. 


\section{ESTUDIOS}

Tabla 4. Niveles de confianza según el alcance del medio

\begin{tabular}{|l|c|c|c|c|}
\hline \multicolumn{2}{|c|}{ Local o regional } & DE & M & DE \\
\hline Nivel de confianza & $\mathrm{M}$ & 1,03 & 2,88 & 0,92 \\
\hline El Parlamento & 2,79 & 0,95 & 2,26 & 1,02 \\
\hline Los partidos políticos & 2,24 & 0,79 & 2,06 & 0,83 \\
\hline Los políticos en general & 1,96 & 0,79 & 2,10 & 0,84 \\
\hline Índice & 2,11 & $\mathbf{0 , 7 0}$ & $\mathbf{2 , 3 3}$ & $\mathbf{0 , 7 2}$ \\
\hline
\end{tabular}

Fuente: elaboración propia.

Nuestros resultados tampoco mostraron diferencias significativas (figura 3), $t(386)=-0,047, p=0,963$, BootCl $_{95}=-0,22$ a 0,21 , entre el nivel de confianza hacia las instituciones de las/los periodistas de medios mayoritariamente públicos o estatales ( $\mathrm{M}=2,29, \mathrm{DE}=0,80$ ) y las/los profesionales de medios mayoritariamente privados $(\mathrm{M}=2,28, \mathrm{DE}=0,68)$. Esta prueba no proporciona evidencia empírica para sustentar la hipótesis 3 . Un examen detallado a cada uno de los indicadores que conforman el constructo central (tabla 5) mostró que tampoco existen diferencias significativas en cada uno de ellos ${ }^{6}$.

6 Sólo en el caso del sistema judicial (incluido en el índice general de los diez indicadores), el nivel de confianza es significativamente mayor, $\mathrm{t}(382)=2,309, \mathrm{p}<0,05, \mathrm{BootCl}_{95}=-0,03$ a $0,57, d=0,33$, en las /los periodistas de los medios privados ( $M=3,00, D E=0,93$ ) en comparación con el de las/los periodistas de los medios públicos ( $M=2,69, D E=0,94)$. El tamaño de estas diferencias sigue siendo bajo. 


\section{ESTUDIOS}

Figura 3. Variación de los niveles de confianza según la propiedad del medio

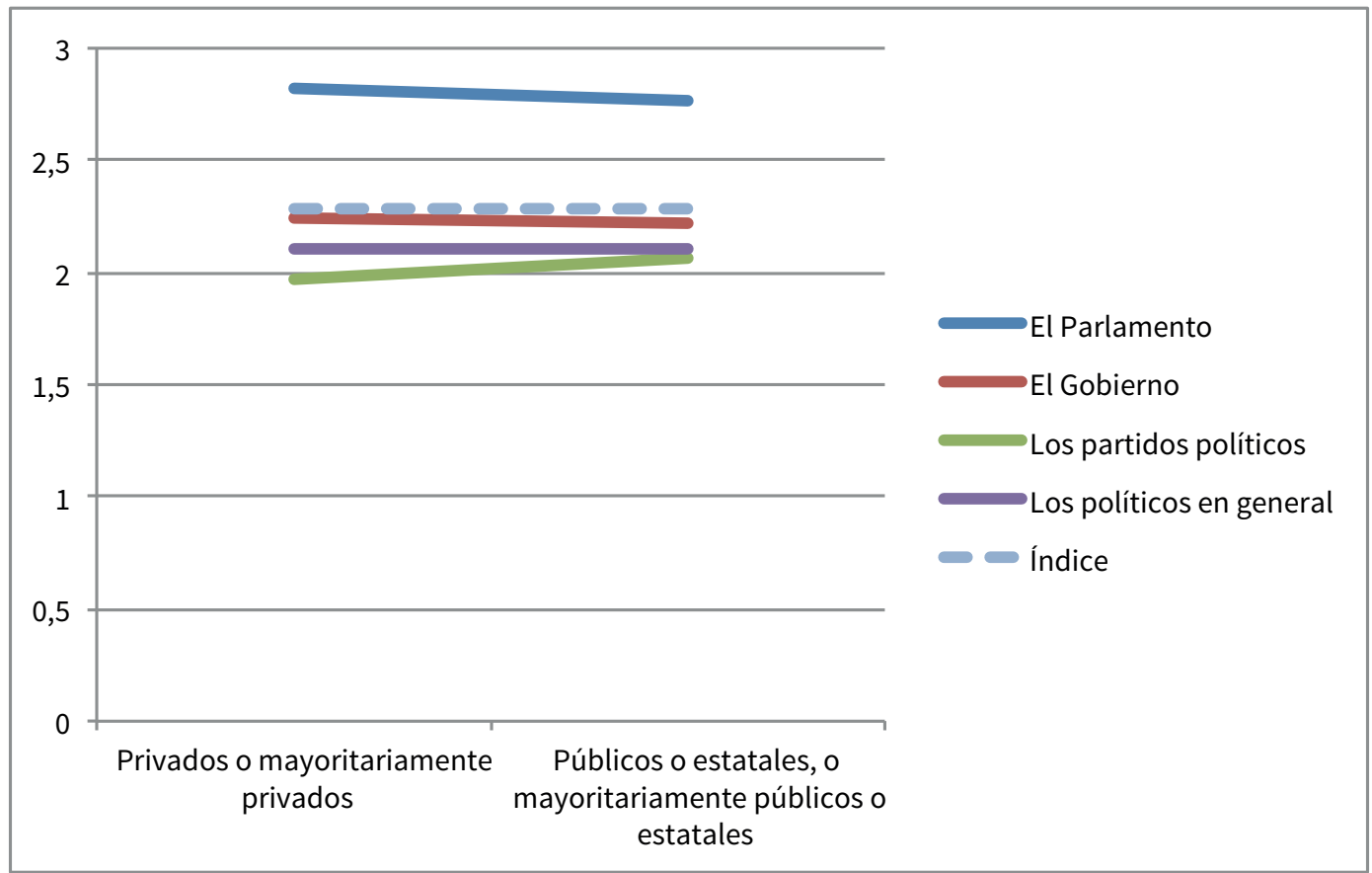

Fuente: elaboración propia.

Tabla 5. Niveles de confianza según la propiedad del medio

\begin{tabular}{|c|c|c|c|c|}
\hline \multirow{2}{*}{ Nivel de confianza } & \multicolumn{2}{|c|}{ Privados o mayoritariamente privados } & \multicolumn{2}{|c|}{$\begin{array}{l}\text { Públicos o estatales, o mayoritariamente } \\
\text { públicos o estatales }\end{array}$} \\
\hline & M & DE & M & $\mathrm{DE}$ \\
\hline El Parlamento & 2,82 & 1,01 & 2,76 & 1,01 \\
\hline El Gobierno & 2,24 & 0,96 & 2,22 & 1,01 \\
\hline Los partidos políticos & 1,97 & 0,78 & 2,07 & 0,90 \\
\hline Los políticos en general & 2,11 & 0,79 & 2,10 & 0,89 \\
\hline Índice & 2,28 & 0,68 & 2,29 & 0,80 \\
\hline
\end{tabular}

Fuente: elaboración propia. 


\section{Discusión y conclusiones}

La confianza de las/los periodistas españoles en las instituciones políticas (Parlamento, Gobierno, partidos políticos y políticas/os en general) se sitúa en un nivel bajo ( $\mathrm{m}=2,29$ dentro de una escala de 1 a 5 , en la que 5 significa confianza muy alta). Sin embargo, existen diferencias notables en la valoración de las distintas instituciones: la mejor valorada es el Parlamento, seguida del Gobierno; y, las que menos, los partidos políticos y las/los políticas/ os en general. El hecho de que estas dos últimas sean las peor valoradas sugiere que la principal fuente de desafección periodística hacia la política parece estar generada por la desconfianza hacia los partidos políticos y las/los mismas/os políticas/os, y que la crisis económica ha puesto aún más de manifiesto la malograda responsabilidad de estos actores y actrices institucionales. Por lo tanto, resulta evidente que las y los periodistas españolas/es confían más en las instituciones políticas que vertebran el sistema democrático constitucional que en los individuos que han asumido la responsabilidad de dirigir y administrar dichos organismos.

Tal como señalan Hanitzsch y Berganza $(2012 ; 2014)$ las/los periodistas presentan un nivel superior de confianza en las instituciones públicas en los países que se comportan mejor en términos de corrupción (2012, p. 806; 2014, p. 146), lo cual tiene sentido también en el caso español. Así, esta pérdida de confianza de las/los periodistas que hemos observado desde 2011 puede deberse al aumento de la percepción de la corrupción que se ha experimentado en los últimos años en España. Tal como apuntábamos ya, según los datos del Barómetro de mayo de 2015 del cıs, la situación política actual es percibida como problemática por la ciudadanía, debido principalmente a la corrupción política. El 42,1\% de las personas encuestadas califica la situación política en España de muy mala, el $34,4 \%$ de mala y el $17,7 \%$ de regular. Solo un $2,7 \%$ la considera buena y un $0,2 \%$ muy buena. Un $50,8 \%$ sitúa la corrupción y el fraude entre los tres primeros problemas que existen actualmente en España; para un $20,9 \%$ es el principal problema y para un 22,5\%, el segundo. Es más, estudiando los datos de manera diacrónica a partir de la evolución del índice de percepción de la corrupción elaborado por Transparencia Internacional, de 2012 a 2014 esta percepción ha aumentado en un 7,69\%.

Si comparamos estos datos con los recogidos en España en 2011 también dentro del marco del estudio Worlds of Journalism (Hanitzsch \& Berganza, 2014), observamos, por un lado, un descenso significativo en la valoración de estas cuatro instituciones políticas. Por otra parte, se verifica la misma tendencia en la valoración de las distintas instituciones: el Parlamento continúa siendo la más valorada, aunque con un descenso significativo de 0,6 puntos (pasa de 3,41 puntos en la escala de 1 a 5, a 2,81). Además, los partidos políticos siguen siendo los menos valorados ( $\mathrm{m}=1,98)$, aunque el descenso prácticamente no es perceptible (en 2011: $\mathrm{m}=2,04)$ ). El verdadero descenso con respecto a 2011 se encuentra en los datos de confianza hacia el Gobierno. Si en 2011 era de $\mathrm{m}=3,24$ ( $3=$ algo de confianza), en 2015 es de 2,24 (2=poca confianza). Así, si en 2011 las/los periodistas mostraban un menor nivel de escepticismo hacia el Parlamento y el Gobierno en comparación con los/as políticos/as y los políticos en general, en 2015 el Gobierno ya no mantiene esa posición de mayor confianza comparativa y se acerca a los niveles de confianza que las/los profesionales de la comunicación otorgan al grupo de las/los políticas/os en general y a los partidos políticos.

Por otra parte, resulta interesante observar la tendencia: los mayores índices de confianza los poseen quienes trabajan en medios audiovisuales (radio y televisión), seguidos de los de medios impresos. Quienes más desconfían, 
especialmente del Gobierno, son las y los profesionales de los medios en línea. Podemos afirmar, por tanto, que las/ los periodistas que trabajan en la red son los más escépticos hacia las instituciones políticas en general y de manera especial hacia el Gobierno. Como se ha señalado ya, estudios anteriores han versado sobre la especificidad del trabajo periodístico en la red, lo cual también queda subrayado en esta investigación, ya que la muestra de medios en línea toma exclusivamente medios nativos digitales y medios que, aunque nacieron como las plataformas digitales de medios convencionales, en la actualidad ya sólo existen en la red. No cabe duda de que la trayectoria laboral de estos/as profesionales, muchos/as de los cuales vieron desaparecer los medios en los que trabajaban como consecuencia de la crisis, tuvieron que poner en marcha sus propios medios y/o se vieron forzados a cambiar de trabajo a causa de los despidos y recortes llevados a cabo en los medios convencionales, puede afectar la confianza que mantienen en las instituciones políticas. Dado que las propias características del periodismo en la red también se manifiestan como específicas (véase, por ejemplo, Quandt, 2008), se considera que esta cuestión debe ser objeto de una investigación más atenta. Asimismo, es preciso profundizar en estos resultados llevando a cabo análisis multivariantes, pues el estudio estadístico efectuado impide conocer si determinadas variables individuales -como, por ejemplo, la edad de las/los profesionales- pueden también incidir, y hasta qué punto, en el hecho de que las/los trabajadoras/es de los medios en línea muestren mayores niveles de desconfianza.

En general, no hemos encontrado diferencias significativas entre las/los periodistas en relación con el alcance y propiedad del medio. En todos ellos el nivel de desconfianza es similar (alto), al igual que sucedía en el estudio de Hanitzsch y Berganza (2012), donde se aseguraba que "había en realidad poca varianza de la confianza de los periodistas en el nivel organizacional" (p. 807) y donde no se podía demostrar una relación significativa entre la confianza y la propiedad privada del medio. Sin embargo, que en España no exista un número relevante de medios de comunicación puramente estatales ${ }^{7}$ y que, por ello, hayamos realizado nuestro análisis agregando los datos de los medios públicos y los estatales a la hora de conocer la confianza de las y los profesionales en las instituciones, también puede explicar la diferencia de nuestros resultados con respecto a otros estudios comparativos de ámbito internacional donde se comprueba que los niveles de confianza de las y los periodistas que trabajan en medios estatales son superiores a aquellos que trabajan en medios públicos y privados (Hanitzsch \& Berganza, 2012).

Nuestro estudio se limita a un acercamiento descriptivo y correlacional de la confianza de las y los periodistas en las instituciones públicas. La investigación futura en el campo puede avanzar hacia modelos más explicativos que permitan conocer más en detalle el origen de la desconfianza, por medio, por ejemplo, de entrevistas en profundidad o grupos de discusión. Asimismo, dado el vasto conocimiento que se está generando en la identificación de factores que pueden modificar los niveles de confianza, también se hace necesario progresar hacia estudios que muestren una síntesis de los avances en el campo, por ejemplo, por medio de meta-análisis. Adicionalmente, el avance teórico en el área conllevará también a la aplicación de técnicas experimentales que permitan comprobar las relaciones de causalidad de las variables que hemos incluido en este estudio.

En cualquier caso, este trabajo enmarcado en un proyecto de alcance mundial pone de manifiesto la importancia que tiene la estandarización de constructos para medir la desconfianza en las instituciones. Por un lado, porque los niveles de validez y fiabilidad muestran un constructo lo suficientemente robusto para poder ser usado

7 En nuestra muestra los medios de comunicación puramente estatales alcanzan el 1,5\%. Cuando se unen los medios estatales y públicos, como se ha referenciado ya, este dato alcanza el 14,9\%. 
en distintos contextos geográficos y culturales; y por el otro, porque estas medidas comunes permitirán a proyectos como wss generar resultados comparativos de gran valor para la comunidad científica. De hecho, estos estudios comparativos pueden determinar si en países con características parecidas a las de España, por ejemplo los del modelo mediterráneo (Hallin \& Mancini, 2001), los resultados tienden a ser similares cuando se los compara con los de otras zonas geográficas o culturalmente cohesionadas.

Asimismo, en tanto que un sistema mediático libre de interferencias gubernamentales se ha situado en otros estudios como una condición principal para índices de confianza más elevados (Hanitzsch \& Berganza 2012, p. 806), parece interesante continuar el estudio de las variables de naturaleza macroexternas, planteando preguntas como: ¿a mayor nivel de presión experimentada por las y los periodistas por parte de las instituciones políticas e individuos, mayor cinismo?, ¿son los factores coyunturales nacionales la clave para explicar el cinismo de las y los periodistas?, ¿en un contexto económico mejor, hay mayor confianza? De este modo, resulta preciso avanzar en el estudio del fenómeno con aproximaciones que pongan en relación la confianza de las y los informadoras/ es con aspectos propios de su ámbito individual, de su cultura profesional y del sistema político-social en el que enmarcan su trabajo. Por otra parte, se precisa determinar (con constructos equivalentes) si la confianza de las y los periodistas en las instituciones es mayor, similar o inferior a los niveles del público en general. Estas cuestiones serán mejor respondidas en la medida en que se planteen y mantengan estudios de tipo longitudinal.

\section{Agradecimientos}

Las autoras y el autor agradecen al Dr. Carlos Arcila Calderón, profesor de la Universidad de Salamanca, la valiosa ayuda prestada en la elaboración de este trabajo a la hora de realizar los análisis estadísticos y su interpretación. También en la realización de distintas encuestas en el marco del trabajo de campo.

\section{Referencias}

1. APM (2013). Informe anual de la profesión periodística 2013. Madrid: Asociación de la Prensa de Madrid.

2. Brants, K., de Vreese, C., Möller, J., \& van Praag, P. (2010). The real spiral of cynicism? Symbiosis and mistrust between politicians and journalists. International Journal of Press/Politics, 15(1), 25-40. Doi: dx.doi.org/10.1177/1940161209351005

3. Cappella, J. N., \& Jamieson, K. H. (1997). Spiral of cynicism: The press and the public good. New York, NY: Oxford University Press.

4. Centro de Investigaciones Sociológicas (CIS) (2015). Barómetro de mayo 2015. Estudio no 3082. Recuperado de http://datos.cis.es/pdf/Es3082mar_A.pdf

5. Cohen, J. (1977). Statistical power analysis for the behavioral sciences. Hillsdale, New Jersey: Lawrence Erlbaum Associates, Inc.

6. Cook, T. E. (1996). Governing with the news: The news media as a political institution. Chicago: University of Chicago Press.

7. Cronbach, L. J. (1951). Coefficient alpha and the internal structure of tests. Psychometrika, 16(3), 297-334. 
8. Díaz Nosty, B. (2011). Libro negro del periodismo en España. Madrid: Cátedra unesco de ComunicaciónUniversidad de Málaga, Asociación de la prensa de Madrid.

9. Donohue, G. A., Olien, C. N., \& Tichenor, P. J. (1985). Reporting conflict by pluralism, newspaper type and ownership. Journalism Quarterly, 62(3), 489-499.

10. Gans, H. J. (2003). Democracy and the News. Oxford: Oxford University Press.

11. Hallin, D. C. \& Mancini, P. (Eds.). (2011). Comparing media systems beyond the western world. New York: Cambridge University Press.

12. Hanitzsch, T., \& Berganza, R. (2012). Explaining journalists' trust in public institutions across 20 countries: Media freedom, corruption, and ownership matter most. Journal of Communication, 62(5), 794-814.

13. Hanitzsch, T., \& Berganza, R. (2014). Political trust among journalists: Comparative evidence from 21 countries. En Canel, M. J. \& Voltmer, K. (Eds.), Comparing political communication across time and space: New studies in an emerging field (pp. 137-156). London: Palgrave McMillan.

14. Hanitzsch, T., \& Mellado, C. (2011). What shapes the news around the world? How journalists in eighteen countries perceive influences on their work. International Journal of Press/Politics 16(3), 404-426.

15. Hayes, A. (2005). Statistical methods for communication science. Mahwah, NJ: Lawrence Erlbaum Associates.

16. Hudson, J. (2006). Institutional trust and subjective well-being across the EU. Kyklos, 59(1), 43-62.

17. Igartua, J. J. (2006). Métodos cuantitativos de investigación en comunicación. Barcelona: Bosch.

18. Macía, F. (2010). Validez de los tests y el análisis factorial: Nociones generales. Ciencia y Trabajo, 12(35), pp. 276-280.

19. Mair, P. (2006). Polity scepticism, party failings, and the challenge to European democracy. Wassenaar: NIAS.

20. McManus, J. H. (1994). Market driven journalism: Let citizen beware? Thousand Oaks, CA: Sage.

21. Mellado, C., \& van Dalen, A. (2014). Between rhetoric and practice: Explaining the gap between role conception and performance in journalism. Journalism Studies, 15(6), 859-878.

22. Mishler, W., \& Rose, R. (2001). What are the origins of political trust? Testing institutional and cultural theories in post-communist societies. Comparative Political Studies, 34(1), 30-62.

23. Misztal, B. (1996). Trust in modern societies: The search for the bases of social order. Cambridge: Polity Press.

24. Pérez-Gil, J., Chacón, S., \& Moreno, R. (2000). Validez de constructo: el uso de análisis factorial exploratorio-confirmatorio para obtener evidencias de validez. Psicothema, 12(2), 442-446.

25. Quandt, T. (2008). No news on the world wide web? A comparative analysis of online news in Europe and the United States. Journalism Studies, 9(5), 717-738.

26. Reich, Z., \& Hanitzsch, T. (2013). Determinants of journalists' professional autonomy: Individual and national level factors matter more than organizational ones. Mass Communication and Society, 16(1), 133-156.

27. Robinson, M. (1976). Public affairs television and the growth of political malaise: The Case of the Selling the Pentagon, American Political Science Review, 70(3), 409-432.

28. Shoemaker, P. J., \& Reese, S. D. (1996). Organizational influences on content. En Mediating the Message: Theories of Influences on Mass Media Content, (pp. 133-165). New York: Longman.

29. Tsfati, Y. (2003). Does audience skepticism of the media matter in agenda setting? Journal of Broadcasting \& Electronic Media, 47(2), 157-176. 


\section{ESTUDIOS}

30. Uriarte, E. (2002). Los medios de comunicación de masas y la opinión pública. En Introducción a la ciencia política. La política en las sociedades democráticas. Madrid: Editorial Tecnos.

31. van Dalen, A.; Albæk, E. \& de Vreese, C. (2011). Suspicious minds: explaining political cynicism among political journalists in Europe. European Journal of Communication, 26(2), 147-162. Doi: dx.doi.org/10.1177/ 0267323111404841

32. Weaver, D., Beam, R. A., Brownlee, B. J., Voakes, P. S., \& Wilhoit, G. C. (2007). The American journalist in the 21st century: U.S. News People at the Dawn of a New Millennium. Mahwah, NJ: Erlbaum.

33. Weaver, D., \& Löffelholz, M. (2008). Questioning national, cultural and disciplinary boundaries: A call for global journalism research. En Global Journalism Research. Theories, Methods, Findings, Future, (pp. 3-12). Oxford: Blackwell.

34. Zhu, J. H., Weaver, D., Lo, V., Chen, C., \& Wu, W. (1997). Individual, organizational, and societal influences on media role perceptions: A comparative study of journalists in China, Taiwan, and the United States. Journalism \& Mass Communication Quarterly, 74(1), 84-96. 